Check for updates

Cite this: RSC Adv., 2019, 9, 28627

\title{
A study on graphene composites for peripheral nerve injury repair under electrical stimulation
}

\author{
Zhiqiang Huang, ${ }^{a}$ Zhenzhao Guo, (D) $^{\mathrm{b}}$ Manman Sun, ${ }^{\text {a Shaomao Fang }}{ }^{\mathrm{a}}$ \\ and Hong $\mathrm{Li}$ (D)*a
}

Electrical stimulation (ES) provides an effective alternative to peripheral nerve repair via conductive scaffolds. The aim of the present study is to investigate a graphene (GR)/thermoplastic polyurethane (TPU) composite for the repair of peripheral nerve injury under ES. To this end, conductive composite membranes were fabricated by blending GR (2, 4 and 6 wt\%) with TPU. GR maintains its own structure in the composite and enhances the mechanical and electrical properties of the composite. The composites with excellent biocompatibility had a hemolysis rate of less than $5 \%$. As a result, the 4GR-TPU (4 wt\% GR) sample with enhanced mechanical properties possessed the highest conductivity value of $33.45 \pm$ $0.78 \mathrm{~S} \mathrm{~m}^{-1}$. Compared with the non-conductive sample, 4GR-TPU was favorable for the viability of Schwann cells (SCs) under ES. When different voltages of ES were applied, a direct current of $10 \mathrm{mV}$ was more suitable for the growth and proliferation of SCs. This study provides beneficial information for peripheral nerve repair via ES.

Received 27th June 2019

Accepted 21st August 2019

DOI: $10.1039 / \mathrm{c} 9 \mathrm{ra0} 4855 \mathrm{c}$

rsc.li/rsc-advances

neurons, suggesting that ES modifies SC phenotype. ${ }^{8}$ Moreover, the better the development of the role of SCs through ES, the better would be the application in the repair of PNI.

Due to the intrinsic electro-activity of nerve cells, ${ }^{9}$ conductive materials were of importance in PNI regeneration by offering ways for applying ES. Conductive biomaterials should provide local ES and templates for nerve cell growth and tissue repair, and allow for the precise external control of stimulation levels and duration. ${ }^{\mathbf{1 0 - 1 4}}$ In order to realize the functions of the materials, combining the polymers with conducting nanoparticles to improve the mechanical support, conductivity and biocompatibility has been one of the strategies. ${ }^{15-18}$ In the past decade, some conductive materials (e.g., polypyrrole (PPY) and polyaniline (PANI)) have shown superior electrical properties in nerve regeneration via ES. ${ }^{19-21}$ However, due to the poor processing performance and (or) biocompatibility, the recovery function of the materials was seriously limited. Most importantly, the resistivity of the conductive scaffolds consisting of these polymer materials is in the range of 1000-40000 $\Omega \mathrm{cm} .^{22-24}$ Therefore, a larger ES intensity may be required, which might damage the neural cells and tissues. An ideal conductive scaffold should provide not only electrical properties but also biocompatibility cues for guiding neural cell growth.

The role of cells in tissue engineering and regeneration still has much space for improvement via the modification of biomaterials. Graphene is a two-dimensional $\mathrm{sp}^{2}$ hybrid carbon monolayer with honeycomb arrangement and high conductivity, mechanical strength, and ultra-high specific surface area as a kind of nanosized conductive filler. Graphene-based
${ }^{a}$ Department of Materials Science and Engineering, Jinan University, China. E-mail: tlihong@jnu.edu.cn

${ }^{b}$ The First Affiliated Hospital of Jinan University, Jinan University, China 
nanomaterials have become biocompatible and electroactive scaffolds for neurogenesis due to their adjustable chemical, physical and mechanical properties. ${ }^{25}$ Most of the materials are graphene films with various surface functionalities to improve the biocompatibility. Meanwhile, a pre-coating layer of either laminin, collagen, poly-L-lysine (PLL), or PDL is generally applied on the graphene film to support the adhesion of the cell. Moreover, a coating layer of graphene is generally applied on composite scaffolds for neural engineering, which basically improves the conductivity of materials. Therefore, the coatings of either biochemicals or graphene nanoparticles are easily removable under the flow of body fluids. In recent years, thermoplastic polyurethane (TPU) has exhibited good biocompatibility and it is also a kind of polymer matrix with good elasticity and affinity to carbon fillers. ${ }^{26}$ Furthermore, graphene nanoparticles can significantly improve the electrical conductivity and mechanical properties of the TPU matrix. Meanwhile, TPU improves the biocompatibility and process ability of graphene, which are promising composite materials that can be further studied for tissue regeneration. ${ }^{27-29}$

Here, a conductive TPU membrane with graphene filler was prepared for PNI regeneration via solvent blending. Subsequently, the structural properties, electrical resistance, mechanical properties and biocompatibility were investigated. The effects of ES on the morphologies and proliferation of SCs with appropriate ES parameter were analysed on a self-made 24well vertical ES platform. With suitable ES parameters, the growth and spread of SCs on the GR-TPU composite films improved. This study can provide more beneficial information on the application of ES for PNI repair.

\section{Experimental}

\subsection{Materials}

Graphene (06-0210) was obtained from Strem Chemicals Corporation. TPU (2363-80AE) was purchased from Lubrizol Corporation. Tetrahydrofuran (THF) was obtained from Sigma, United Kingdom. Rat Schwann cells (RSC-96) were purchased from Beijing DingGuo biotechnology Co. Deionized water was used throughout the experiments.

\subsection{Preparation of graphene-TPU composite membranes}

For the obtained GR-TPU composite membranes, $0.02 \mathrm{~g}, 0.04 \mathrm{~g}$ and $0.06 \mathrm{~g}$ of graphene nanoparticles were dispersed in $20 \mathrm{~mL}$ of THF, respectively, stirred for $30 \mathrm{~min}$ to obtain different concentrations of graphene dispersion, and sonicated for $3 \mathrm{~h}$ to minimize the aggregation of graphene particles. Simultaneously, $1.00 \mathrm{~g}, 0.98 \mathrm{~g}, 0.96 \mathrm{~g}$ and $0.94 \mathrm{~g}$ of TPU were dissolved in $15 \mathrm{~mL}$ of THF, respectively, and stirred for $12 \mathrm{~h}$ at a rotational speed of $500 \mathrm{rpm}$ at room temperature. Then, the graphene dispersion was added into the TPU solution to set the graphene contents at 2, 4, and $6 \mathrm{wt} \%$ in TPU-based composites, respectively. After sonication for $1 \mathrm{~h}$, the resultant dispersions were noted as 2GR-TPU, 4GR-TPU and 6GR-TPU for the graphene concentrations of 2,4 , and $6 \mathrm{wt} \%$, respectively. The membranes were obtained by the solution casting method in which the GR-
TPU composite solution was poured onto a polytetrafluoroethylene (PTFE) dish that was dried at room temperature for $12 \mathrm{~h}$ and vacuum dried for another $12 \mathrm{~h}$. The pure TPU membrane was also prepared by following the same steps.

\subsection{Characterization of composite membranes}

2.3.1. Observation of morphology. The GR-TPU composite membranes were coated with gold (SBC-12, KYKY, China), and the surface morphology was studied using a scanning electron microscope (XL30 FESEM, Philips, Netherlands) at a magnification of $10000 \times$.

The size and structure of graphene nanosheets in TPU membranes were observed by transmission electron microscopy (TEM, Tecnai G2 F30, FEI) under $120 \mathrm{kV}$. Samples for TEM observation were prepared by ultracryotomy (Leica FC7).

2.3.2. Hydrophilicity test. The water contact angle of the GR-TPU membranes was measured by the distilled water static drop method, and the surface hydrophilicity of the GR-TPU membrane was studied (DSA-100, Kruss, Germany).

2.3.3. Chemical structural analysis. Fourier transform infrared (FTIR) measurements of GR-TPU systems were performed (iS10, Nicolet, USA) in the range of $3800-600 \mathrm{~cm}^{-1}$ at room temperature. X-ray diffractometer (XRD) using $\mathrm{Cu} \mathrm{K} \alpha$ radiation (MiniFlex 600, Rigaku, Japan) was employed to obtain the XRD patterns of GR-TPU membranes. The groups were scanned over the $2 \theta$ range from $5^{\circ}$ to $60^{\circ}$ at a rate of $5^{\circ}$ per minute.

2.3.4. Thermodynamic properties. The thermodynamic properties of GR-TPU membranes were determined by differential scanning calorimetry (DSC) using a thermal analysis apparatus (Q20, TA, USA). Each group of about $5 \mathrm{mg}$ samples were put into an alumina crucible. The initial temperature was set as $50{ }^{\circ} \mathrm{C}$. First, all the experiments were carried out at a flow rate of $20 \mathrm{~mL} \min ^{-1}$ in a nitrogen atmosphere. Then, the membranes were heated to $210{ }^{\circ} \mathrm{C}$ at a heating rate of $10{ }^{\circ} \mathrm{C} \mathrm{min}{ }^{-1}$ and held isothermally for $3 \mathrm{~min}$ to eliminate the thermal history, after which the samples were cooled to $-70{ }^{\circ} \mathrm{C}$ and reheated to $210{ }^{\circ} \mathrm{C}$ at a rate of $10{ }^{\circ} \mathrm{C} \mathrm{min}{ }^{-1}$.

2.3.5. Electrical characterization. The resistivity of composite membranes under dry conditions was measured at room temperature by the four-probe method using a Film Block Resistance Tester (KDB-1, JSS, China). Six duplicates were used for each test. The resistivity tested here is the square resistance mode, which means that the material is cut into squares of $1 \times$ $1 \mathrm{~cm}$ dimension.

The electrical conductivity $(S)$, in $\mathrm{S} \mathrm{m}^{-1}$, was calculated using the following equation:

$$
S=\frac{1}{\rho} \times 100, \text { in } \mathrm{S} \mathrm{m}^{-1} .
$$

where $\rho=$ electrical resistivity in $\Omega \mathrm{cm}$.

\subsection{Evaluation of mechanical property}

The mechanical properties of composite membranes were determined using a Universal Testing Machine (UTM-1422, JJTEST, China), by measuring the length, width and thickness 
of the samples. A $100 \mathrm{~N}$ load cell was used at room temperature at a speed of $20 \mathrm{~mm} \mathrm{~min}^{-1}$. After the tensile process, the stressstrain curves of the specimens were obtained. From the stressstrain curves of the specimens, the corresponding mechanical parameters were obtained: elongation at break, tensile strength and tensile modulus.

\subsection{Hemolysis}

Herein, $5 \mathrm{~mL}$ fresh anticoagulant whole blood and physiological saline were added into a centrifugal tube. The anticoagulant whole blood was washed 2-3 times with physiological saline by centrifugation at $1500 \mathrm{rpm}$ for $15 \mathrm{~min}$, and then the red blood cells were centrifuged. More physiological saline was added to adjust the red blood cell concentration to $2 \% .5 \mathrm{~mL}$ of diluent was added to a $10 \mathrm{~mL}$ centrifuge tube and $1 \times 1 \mathrm{~cm}$ membrane samples were added. The group in which the solvent was ultrapure water was used as the positive control group, and that in which physiological saline was used as the solvent served as the negative control group. These tubes were incubated at $37^{\circ} \mathrm{C}$ for $2 \mathrm{~h}$. Then they were centrifuged at $1500 \mathrm{rpm}$ for $5 \mathrm{~min}$, and the absorbance was measured at $540 \mathrm{~nm}$ using an ELISA reader. The hemolysis rate was calculated according to the following formula:

$$
\text { Hemolysis rate }=\frac{A_{1}-A_{3}}{A_{2}-A_{3}} \times 100 \%
$$

where $A_{1}, A_{2}$, and $A_{3}$ were the absorbance of the sample group, the positive control group, and the negative control group, respectively.

\subsection{Behavior of SCs on GR-TPU membrane in vitro}

2.6.1. SC culture. The SCs were cultured in DMEM supplemented with $10 \%$ fetal bovine serum (FBS) and 1\% penicillin-streptomycin in an incubator at $37^{\circ} \mathrm{C}$ in an atmosphere of $5 \% \mathrm{CO}_{2}$. Taking into account the excellent electrical and mechanical properties of the 4GR-TPU membrane, subsequent works used the 4GR-TPU membrane. The samples of the 4GR-TPU membrane, pure TPU membrane (positive control) and cover slip (negative control) were sterilized with $75 \%$ ethanol for $30 \mathrm{~min}$ and irradiated with UV light for $2 \mathrm{~h}$. Then, SCs were seeded at a density of 5000 cells per well on the membranes.

2.6.2. Electrical stimulation. For ES treatment, the sample was cut into a disc of about $18 \mathrm{~mm}$ in diameter. ES was performed after SC seed plating for 12 hours, and the SCs were stimulated under 10, 50, $100 \mathrm{mV}$ DC voltage and an ES time of 1 hour per day (for a total of 5 days). The electrodes were pressed tightly at the ends of the materials during ES. During the experiment, the medium used for culture SCs was replaced with fresh medium after every electrical stimulation. ${ }^{30,31}$ The selfmade plate and the ES device are shown in Fig. 1. The 24-well plate cover and the electrode were self-made, and the DC power supply was from a double display potentiostat (DJS-292, INESA instrument, Shanghai).

2.6.3. Biocompatibility characterization under ES in vitro. After the evaluation of the physiochemical properties, 4GR-TPU
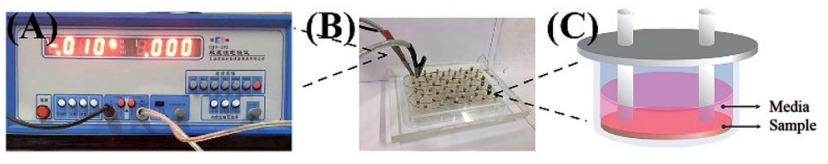

Fig. 1 Self-made electrical stimulation device fitted for 24-well cell culture plate. (A) DC power supply, (B) electrical stimulation device and (C) schematic diagram of electrical stimulation device.

was selected as a scaffold for the ES study. ES with different voltages of $10 \mathrm{mV}, 50 \mathrm{mV}$ and $100 \mathrm{mV}$ was applied on 4GR-TPU membranes. The proliferation of SCs on 4GR-TPU membranes cultured under ES was measured for 1, 3 and $5 \mathrm{~d}$ after cell seeding by an MTT assay (Cytation 3, USA). Fresh DMEM with $10 \%$ MTT solution was added. After $4 \mathrm{~h}$ of incubation, the medium was replaced with $500 \mu \mathrm{L}$ of dimethyl sulfoxide (DMSO) and shaken for $10 \mathrm{~min}$. Then, $100 \mu \mathrm{L}$ of the solutions were transferred into a 96-well plate, and the absorbance was measured at $490 \mathrm{~nm}$ using an ELISA reader. The result of each experiment was reported as mean $\pm \mathrm{SD}$.

Cytotoxicity can be detected by the acridine orange/ethidium bromide ( $\mathrm{AO} / \mathrm{EB})$ dual fluorescence staining method, and the ratio of living cells to dead cells can be calculated. When SCs were cultured on 4GR-TPU membranes for 3 days at different voltages, the culture medium was discarded. $1 \mathrm{~mL}$ of working fluid containing $20 \mu \mathrm{L}$ of AO/EB mixture was added. After 5 minutes, the SCs were washed 2-3 times with PBS. The SC morphology was observed and photographed using an inverted fluorescence microscope (XDY-2, China).

To study the effect of ES on SCs on the conductive or nonconductive membranes, an ES of $10 \mathrm{mV}$ was applied on TPU and 4GR-TPU membranes. The proliferation of SCs on 4GRTPU and TPU membranes cultured under ES was measured for 1, 3 and $5 \mathrm{~d}$ after cell seeding by an MTT assay. The result of each experiment was reported as mean $\pm \mathrm{SD}$.

2.6.4. Cell morphology under ES. In order to observe the cell morphology, the samples were washed 2-3 times with PBS to remove the unviable cells and residual medium, and fixed in $4 \%$ paraformaldehyde. The SCs on the 4GR-TPU membranes were dehydrated with a gradient ethanol solution $(30 \%, 50 \%$, $70 \%, 80 \%, 90 \%, 95 \%$, and $100 \%$ ) and then dried for $24 \mathrm{~h}$. Before the observation, the samples were sputter coated with gold, and the SC morphology on the 4GR-TPU membranes was investigated at an accelerating voltage of $15 \mathrm{kV}$ by SEM (TM3030, Hitachi, Japan). The pictures were taken at $\times 1000$ magnifications.

The microstructures and skeleton of the SCs were observed using a laser scanning confocal microscope (LSCM, ZeissLSM510, Germany). The samples were fixed with $4 \%$ paraformaldehyde, and then washed three times with PBS and permeabilized with $0.5 \%$ Triton X-100 for $10 \mathrm{~min}$. After washing 3 times with PBS, $5 \mu \mathrm{L}$ fluorescein isothiocyanate (FITC) and 500 $\mu \mathrm{L}$ PBS were mixed as the working solution, and the SCs were incubated with FITC for $40 \mathrm{~min}$ and washed 3 times with PBS. Then, the 4,6-diamino-2-phenyl indole (DAPI) working solution was prepared $(1: 1000)$. The SCs were incubated with DAPI for 
$10 \mathrm{~min}$ and then washed three times. The dyeing process was carried out in a dark environment.

\subsection{Statistical analysis}

Electrical conductivity, mechanical property, hemolysis, and MTT data were recorded as mean \pm standard deviation. The MTT results were repeated three times. The analysis of variance (ANOVA) was used to determine the statistical significance of each group. A $p$-value less than 0.05 was considered as statistically significant.

\section{Results}

\subsection{Physiochemical properties of GR-TPU composite}

3.1.1. Morphology. As shown in Fig. 2A, the morphology of GR-TPU membrane and the dispersion of graphene in TPU were observed by FESEM. It could be seen from the plane diagram that the surface of the membranes became rough and wrinkled with the increase in the graphene content. When the content of graphene reached $6 \%$, graphene nanosheets were easy to accumulate, which made the surface rough.

The TEM results of 4GR-TPU membrane are shown in Fig. 2B. It can be seen that most of the graphene nanosheets were uniformly dispersed in TPU. The size of graphene sheets was mostly about 1-2 $\mu \mathrm{m}$ in length and $200 \mathrm{~nm}$ in thickness. The graphene were in the form of a nanosheet with multilayers in the local enlarged image. The results kept accord with other reports. ${ }^{28,32,33}$

3.1.2. Surface hydrophilicity. As shown in Fig. 2C, the contact angle of GR-TPU composite membranes were measured. The surface contact angle of pure TPU membrane was $97.95 \pm 1.25^{\circ}$. For GR-TPU samples, the contact angle decreased gradually with the increase in the graphene concentration. Water droplets on rough surfaces create air cushions in uneven gaps, increasing the contact area between water droplets and air, and thus increasing the wettability of the film surface. ${ }^{34}$ Therefore, the addition of graphene improved the hydrophilicity of the membrane surface. The smaller the contact angle, the higher the surface energy of the material and stronger the interaction between the material and another substance.

3.1.3. Chemical structural analysis. As can be seen from Fig. 2D, in the $110-1078 \mathrm{~cm}^{-1}$ range, the characteristic absorption band of ether was caused by the asymmetric tensile vibration of $\mathrm{C}-\mathrm{O}-\mathrm{C}$. The band at $3330 \mathrm{~cm}^{-1}$ represents the existence of $\mathrm{N}-\mathrm{H}$ tensile vibration. The peaks at 1705 and $1730 \mathrm{~cm}^{-1}$ are due to the tensile vibration of carbonyl groups. The existence of carbonyl group and $\mathrm{N}-\mathrm{H}$ group vibrations proves the existence of the polyurethane group. The bands at 2940 and $2858 \mathrm{~cm}^{-1}$ can be attributed to the asymmetric and symmetric vibrations of $\mathrm{CH}_{2}$ group, respectively. Stretching of the $\mathrm{C}-\mathrm{C}$ bonds within the aromatic ring absorbs radiation in the range of $1600-1585 \mathrm{~cm}^{-1}$ and $1480-1400 \mathrm{~cm}^{-1}$. The stretching vibration of $\mathrm{C}-\mathrm{N}$, in turn, absorbs the radiation at wavenumber $1225 \mathrm{~cm}^{-1}$. The band at $1530 \mathrm{~cm}^{-1}$ can be assigned to the bending vibrations of the $\mathrm{N}-\mathrm{H}$ group. There is no significant shift in the location of vibration bands in the samples modified with graphene. It can be shown that the addition of graphene did not change the chemical structure of TPU. The structural characterization of the composite membrane was carried out by XRD and the plots are shown in Fig. 2E. The XRD pattern of TPU showed the amorphous nature of the polymer with broad peaks at about $20^{\circ}$. The peak corresponding to graphene (the characteristic 002 plane of graphene) had been reported to appear at the $2 \theta$ value of $26^{\circ}$. In addition, as the concentration of
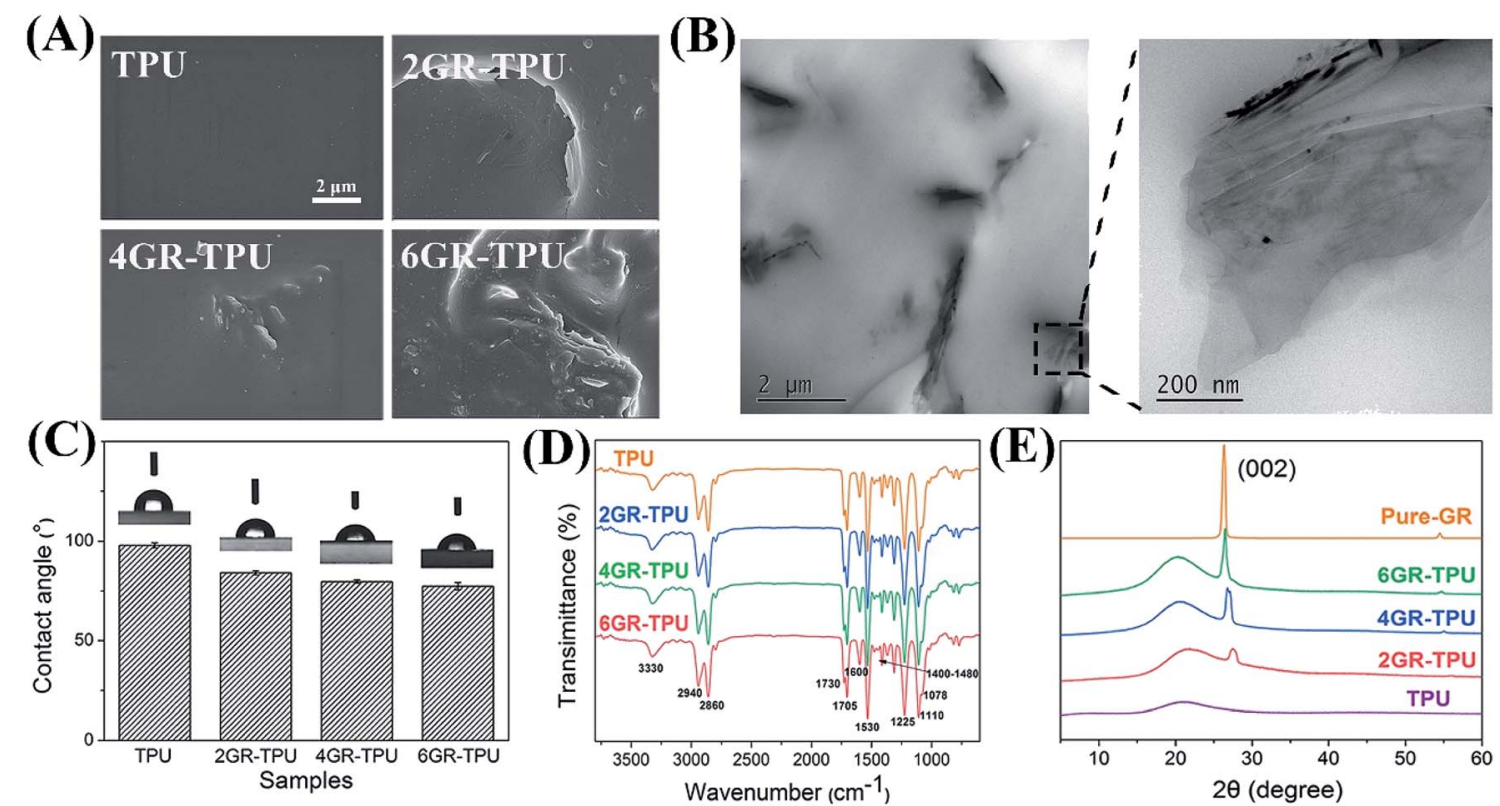

Fig. 2 (A) SEM images of GR-TPU membrane. (B) TEM images of 4GR-TPU membrane. (C) Contact angle histogram. (D) ATR-FTIR spectra and (E) XRD plots of the GR-TPU membranes. 
graphene increased, the characteristic peak had a tendency to become more incisive. This proves that graphene has a regular monolayer structure after blending with TPU.

3.1.4. DSC analysis. DSC experiment was carried out to study the effect of graphene doping on the thermal properties of TPU, and the results are shown in Fig. 3. Compared with pure TPU, both $T_{\mathrm{g}}$ and $T_{\mathrm{m}}$ decreased with the increase in the graphene content. For 6GR-TPU, the temperature of $T_{\mathrm{g}}$ and $T_{\mathrm{m}}$ decreased to about 3.47 and $7.58{ }^{\circ} \mathrm{C}$, respectively. Graphene restricted the mobility of polymer chains, which resulted in the formation of imperfect crystals during crystallization. Therefore, with the increase in the graphene content, the $T_{\mathrm{m}}$ value of the composites tended to decrease. In addition, if the conductive fillers in TPU made the entangled molecular chains of the soft segment looser, then the soft segment in TPU would become easier to move and thus $T_{\mathrm{g}}$ would be reduced.

3.1.5. Electrical conductivity analysis. Table 1 shows the conductivity of GR-TPU membranes. The results indicated that the conductivity of the membranes increased substantially with the addition of graphene. The conductivity of pure TPU membrane exceeded the range of measurement, and the conductivity of 4GR-TPU membrane was the best, which was $33.45 \pm 0.78 \mathrm{~S} \mathrm{~m}^{-1}$. According to our knowledge, the conductivity was higher by more than an order of magnitude compared with other related reports; for example, the surface resistivity of PPY-PLGA prepared by Jae Y. Lee et al. was $10^{3}-10^{4} \Omega$ per square, ${ }^{23}$ while the electrical conductivity of the TPU/rGO nanocomposite prepared by $\mathrm{W}$. Wei et al. increased only from $3.2 \times 10^{-12}$ to $7.8 \times 10^{-7} \mathrm{~S} \mathrm{~m}^{-1} \cdot{ }^{35}$ However, the conductivity of 6GR-TPU membranes showed a downward trend, which may be due to the aggregation of graphene.

\subsection{Mechanical properties analysis}

As can be seen from Fig. 4A, the elongation at break increased from $716.03 \pm 44.13 \%$ for TPU samples to $909.70 \pm 66.11 \%$ for $4 \mathrm{GR}-\mathrm{TPU}$ samples, and decreased for the 6GR-TPU group. A similar effect rule of the tensile strength is shown in Fig. $4 \mathrm{~B}$, and the highest value of tensile strength was $21.78 \pm 3.99 \mathrm{MPa}$ for 4GR-TPU samples. This might be attributed to the interaction between TPU and graphene, which restricted the movement of the polymer chains. When the graphene content reached $6 \%$, the elongation at break and tensile strength of the membranes
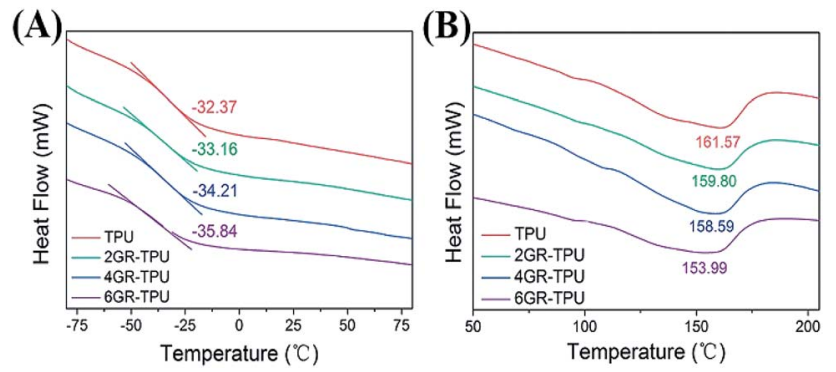

Fig. 3 (A) Glass transition curves and (B) melting curves of GR-TPU composite membranes. decreased, which may be due to the aggregation of graphene, as the modulus is insensitive to the structure of the composite, and mainly dependent on the content of the component. Therefore, the tensile modulus increased from $11.33 \pm$ $0.82 \mathrm{MPa}$ for pure TPU to $15.83 \pm 3.44 \mathrm{MPa}$ for 6GR-TPU, as shown in Fig. 4C.

As shown in Fig. 4D, the typical strain-stress curves of the samples were similar, which proved that the addition of graphene did not change the structure of TPU, which was consistent with the IR result.

\subsection{Hemolysis rate}

Fig. 5 shows the hemolysis rates of different samples in vitro. The data in the histogram showed that the hemolysis rate of the materials was between $0.41 \%$ and $0.51 \%$, which met the international standard of hemolysis of medical biomaterials of less than $5 \%$. The results indicated that GR-TPU membranes had almost no hemolytic reaction in vitro, and red blood cells could maintain its integrity.

\subsection{Effect of ES on cell proliferation and morphology}

In order to investigate the effect of voltage on cell proliferation, the proliferation of SCs on 4GR-TPU membrane was carried out as shown in Fig. 6. As shown in Fig. 6A and B, the ratio of living cells was much greater than the ratio of dead cells no matter how much the voltage was applied. In addition, since the ratio of living cells were almost the same under different voltage conditions, we believe that the appropriate range of ES parameters had little or no cytotoxicity to SCs. As can be seen from Fig. 6C, the cell proliferation was normal under different conditions of ES. Moreover, the proliferation of SCs under $10 \mathrm{mV}$ is the greatest than the groups without ES and other applied voltage. At the voltage of $100 \mathrm{mV}$, the cell proliferation rate was lower than those of other groups. We consider that the excessive voltage would inhibit the SC proliferations, and appropriate ES parameters would have a greater impact on cell growth and proliferation. Therefore, based on the results shown in Fig. 6, the voltage of $10 \mathrm{mV} \mathrm{DC}$ was chosen for further study.

In order to evaluate the SC behaviour on the conductive material, a cover slip and pure TPU samples were selected to co-culture SCs under ES. As shown in Fig. 7, there was no significant difference in cell proliferation on the first day under ES. Moreover, under the condition of no ES, the proliferation of cells on the cover slip was better. However, when the ES of $10 \mathrm{mV}$ DC was applied, the proliferation of SCs on the 4GR-TPU membrane was the highest among all the groups. The data indicated that SCs had better proliferation on the 4GR-TPU membrane under $10 \mathrm{mV}$ DC conditions, that is, an appropriate ES condition could promote the proliferation of SCs.

The SEM images also showed the growth of SCs with ES in Fig. 8. First, it can be seen that cells grew normally on the different materials under ES conditions. Moreover, synaptic stretching was more pronounced under ES. To further investigate the characteristics of the SCs cultured on the membranes 
Table 1 Electrical resistivity of GR-TPU membranes

\begin{tabular}{|c|c|c|c|c|}
\hline Sample & TPU & 2GR-TPU & 4GR-TPU & 6GR-TPU \\
\hline Resistivity $/ \Omega \mathrm{cm}$ & Overload & $9.58 \pm 0.32$ & $2.99 \pm 0.07$ & $3.83 \pm 0.06$ \\
\hline Conductivity/S m ${ }^{-1}$ & Overload & $10.43 \pm 0.35$ & $33.45 \pm 0.78$ & $26.11 \pm 0.41$ \\
\hline
\end{tabular}
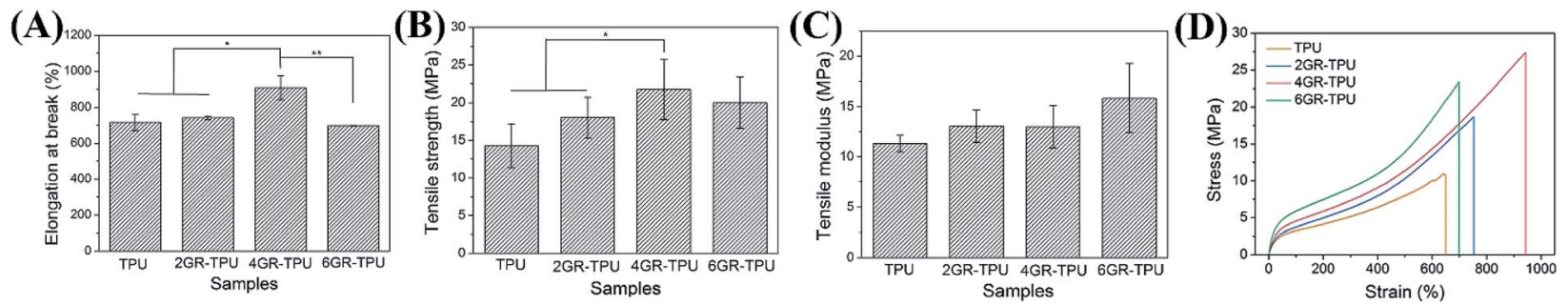

Fig. 4 (A) Mechanical properties of GR-TPU membranes: (A) histogram for ultimate elongation, (B) tensile strength, (C) tensile modulus, and (D) typical stress-strain curves. ${ }^{*}$ Indicates significant difference of $p<0.05 . * *$ Indicates significant difference of $p<0.01$.

under ES with different voltages, LSCM images were obtained (displayed in Fig. 9). It can be seen that the SCs spread well in the presence of $10 \mathrm{mV}$ DC and displayed a cytoskeleton and a well-stretched actin bundle, and many cells grew pseudopods that were similar to those without ES. It proved that the $10 \mathrm{mV}$ DC condition exhibited no or little negative effects on cell spreading. Meanwhile, most of the cells were in a contracted state, spreading poorly in the presence of ES of $100 \mathrm{mV}$ DC. Comparatively, the morphologies of SCs on the cover slip and TPU were similar under the ES of either $100 \mathrm{mV}$ or $10 \mathrm{mV}$. All the results suggested that the $4 \mathrm{GR}$-TPU membrane is suitable for supporting the proliferation of SCs under the ES of $10 \mathrm{mV}$ DC.

\section{Discussion}

Current treatment options for PNI repair are limited and largegap injuries typically do not regain full function, motivating the development of new therapies to promote nerve re-growth. The noted importance of SC participation in PNI repair make SCs an attractive addition to artificial nerve grafts. ${ }^{36}$ Our goals ought to evaluate the sensitivity of SCs to electrical cues in vitro that could better promote axonal growth and other related neural behaviors for PNI repair. By preparing a conductive graphenebased composite membrane with excellent electrical and mechanical property, we demonstrated that SC proliferation can be prompted through appropriate ES. In addition, excessive voltage conditions during ES period repressed the growth and proliferation of SCs.

For electrical and mechanical properties, the properties of composite materials increased when graphene was filled and then decreased when the graphene concentration reached $6 \mathrm{wt} \%$. This phenomenon has been found in many studies..$^{27,37}$ With respect to the mechanical properties, there exists a threshold upon the addition of the graphene contents. Lower than this content, the exfoliated graphene can be well dispersed in the TPU, and an increase in the graphene content can improve the mechanical properties. However, when the graphene content is higher than the threshold of the GR-TPU membrane, it may lead to the stacking of nanosheets and weaken the mechanical properties of GR-TPU membrane. Similarly, for the electrical properties, this phenomenon may be caused by the tunnel resistance between the graphene nanoparticles. ${ }^{38}$ As more graphene contents were added to the matrix, the current passed through with lower resistance, leading to lower resistivity. However, agglomeration may occur when the concentration of graphene reaches a certain level, resulting in no increase in the inter particle gap, which leads to an increase in the tunnel resistance. It can be explained that the mechanical property and resistivity exceed the threshold value with the addition of graphene.

There are different results about the ES affecting the proliferation of SCs. Some studies believe that ES can promote the proliferation of SCs; for example, B. Sun et al. suggested

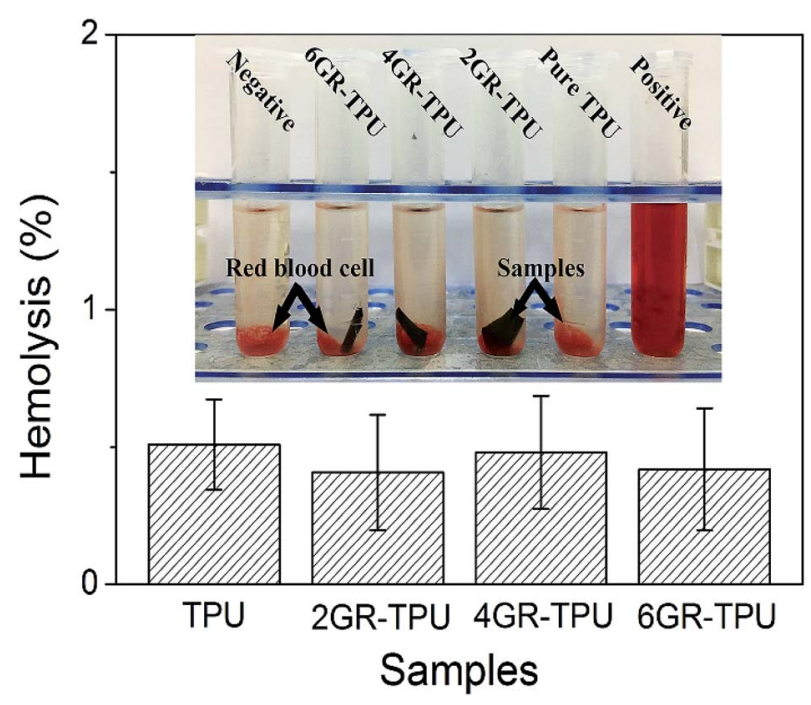

Fig. 5 Hemolytic test of GR-TPU membranes. 

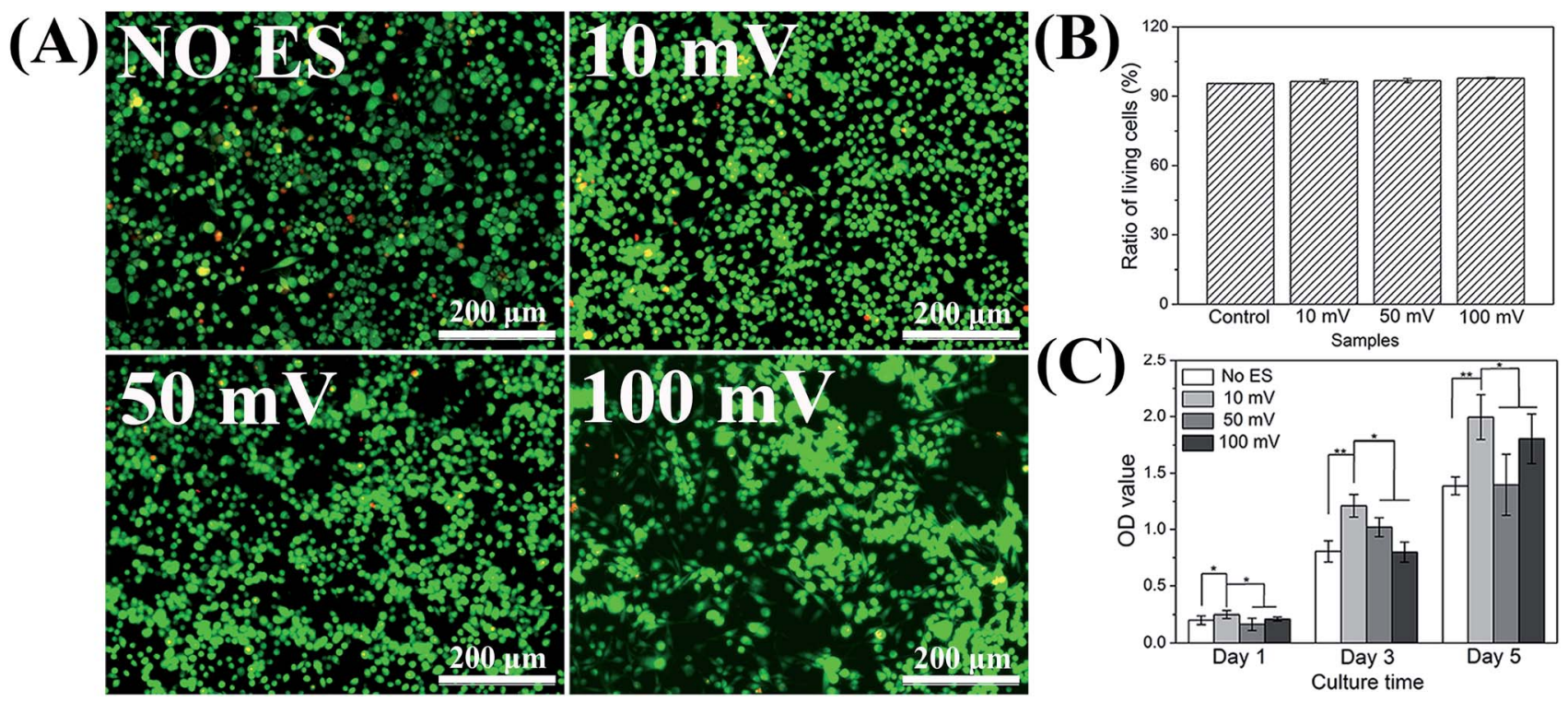

Fig. 6 (A) Fluorescence micrograph of SCs stained with AO/EB on the $3^{\text {rd }}$ day. (B) Ratio of living cells on the 4GR-TPU membrane. (C) MTT result for SCs proliferation with different voltage.

that ES could promote SC proliferation according to the membranes coated with PPY..$^{24,39,40}$ However, in other studies, Abigail N. Koppes et al. suggested that ES had no significant influence on SC proliferation. ${ }^{8}$ Therefore, there was discrepancy on the effect of ES on SC proliferation. In this study, ES with suitable parameters can promote the proliferation of SCs. Many researches have tried to explain the role of ES in nerve regeneration..$^{41,42}$ At present, the problem about the complex mechanism of ES in nerve repair has not been solved absolutely, because the response of cells to ES is not only complicated of cell signal transduction pathways, but also complexity of electrical field in biological media. We believe that a physiological level of ES is effective for activating voltage-sensitive proteins such as ion transmembrane transport and transmembrane receptors, and affects gene expression and cell communication. ${ }^{20}$ Cellular activity is regulated by the distribution of soluble ions through various ion channels, pumps and transporters, which are mainly affected by external stimuli, including ES. ${ }^{43}$ In addition, some membrane proteins regulate cell function through an external electric field. Transient or gated currents may also be affected by external ES. ${ }^{44}$ Therefore, it is supposed that appropriate ES parameters can promote the proliferation of nerve cells and can help the PNI repair.

In this study, the results suggested that 4GR-TPU membrane with $10 \mathrm{mV}$ DC is suitable for SCs to attach and proliferate. DC is widely used in the study of nerve repair, and the magnitude of the voltage has a certain difference on nerve cells. $^{31,45}$ The stimulation evaluated in this study $(0-100 \mathrm{mV}$ DC) $1 \mathrm{~h}$ per day lies within the physiological range, referring to similar investigations. ${ }^{46-48}$ As we know, the intensity of ES has a dual effect on nerve repair. On the one hand, it can accelerate the regeneration of nerve fiber. On the other hand, excessive electric current can damage the nerve regeneration. Although, the mechanism of the effect of current intensity on nerve regeneration has not been fully elucidated, it may be that the strong current intensity makes the internal and external ions unbalanced and changes the normal neural electrophysiological environment, thereby leading to nerve injury. Therefore, the $10 \mathrm{mV}$ DC we chose may be a suitable electrical stimulus for the 4GR-TPU membrane.

However, theranostic application of any nanomaterials warrants great care to ensure that its toxicities are well characterized. The toxicity of graphene and its derivatives mainly depends on their physical and chemical properties, such as shape, size and surface functional groups. ${ }^{49-51}$ There are contradictory results in related studies on the biological safety of graphene and its derivatives. For example, the smaller graphene oxide (GO) $(160 \pm 90 \mathrm{~nm})$ induced decreased cell viability

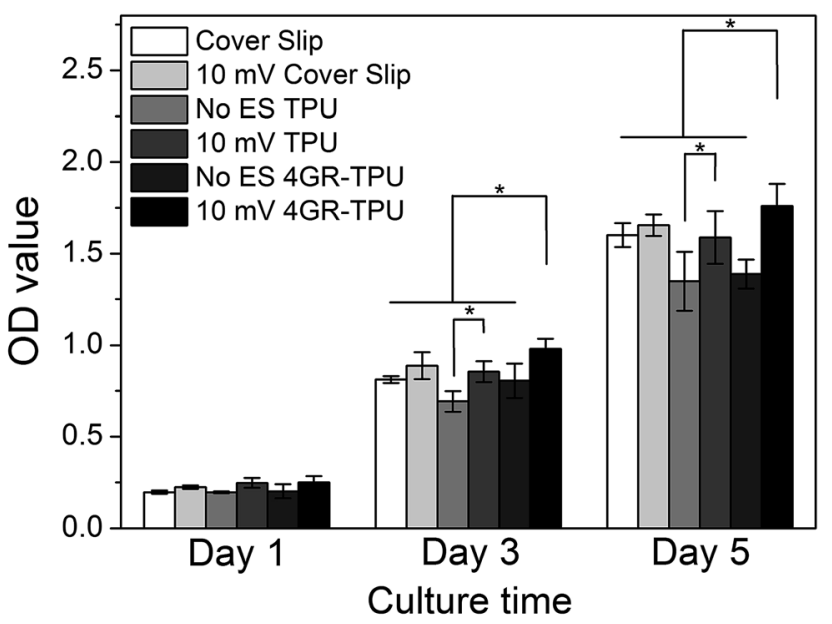

Fig. 7 MTT result for SC proliferation with the ES of $10 \mathrm{mV} \mathrm{DC}$ on different membranes. 


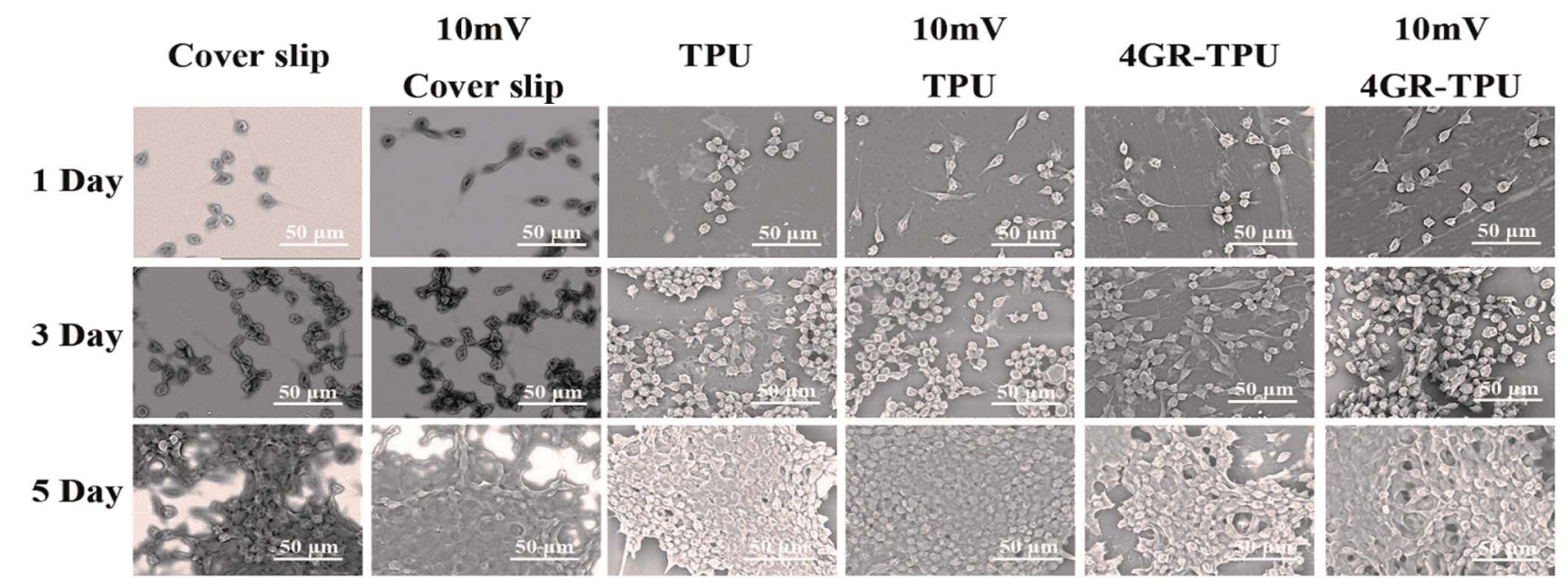

Fig. 8 SEM for SC proliferation with $10 \mathrm{mV}$ DC on different samples.

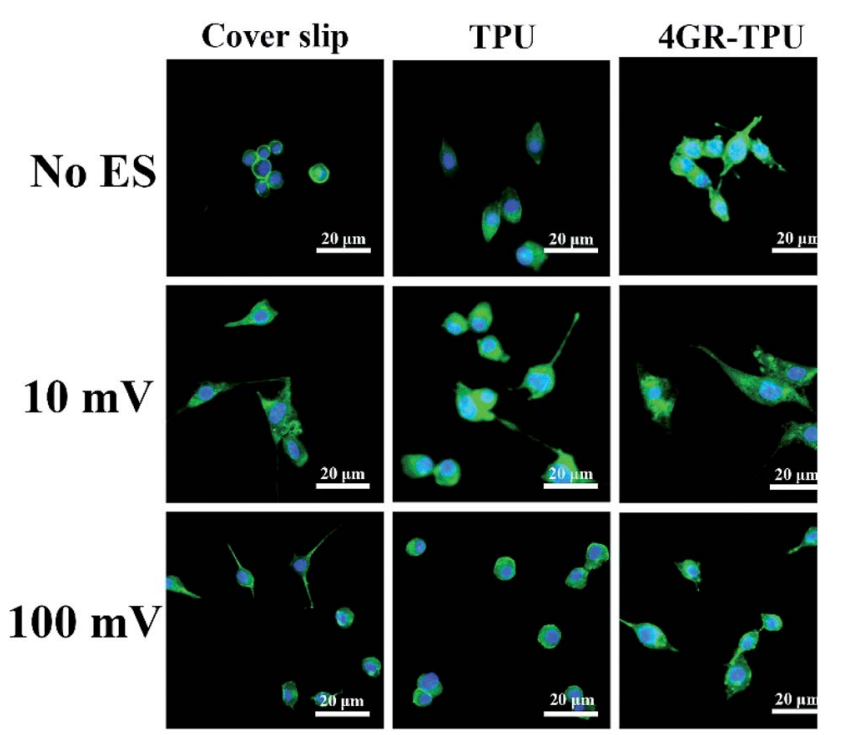

Fig. 9 LSCM for SC proliferation with the ES of $10 \mathrm{mV}$ and $100 \mathrm{mV}$ DC on the $2^{\text {nd }}$ day.

at a higher concentration $\left(200 \mu \mathrm{g} \mathrm{mL}^{-1}\right)$ compared to the larger one $(780 \pm 410 \mathrm{~nm}) .{ }^{52}$ It was also reported that the smaller one has highly potent hemolytic activity, whereas the aggregated rGO showed lesser hemolytic potential. ${ }^{53}$ In this study, although there are no obvious decreased cell viability and high hemolytic activity, the limitations in the absence of corresponding in vivo biological toxicity assessment still exist. Therefore, the graphene-based scaffolds will be further developed in vivo in the future work.

\section{Conclusions}

In this study, conductive TPU membranes with different contents of graphene were obtained by the solution casting method, referred to as GR-TPU. The conductivity and mechanical property could be improved with the addition of graphene. 4GR-TPU with the graphene concentration of $4 \mathrm{wt} \%$ showed a significant enhancement in conductivity. The roles of ES cues in affecting the SC proliferation on the conductive material were investigated. In vitro cell experiments revealed that appropriate ES conditions promote cell growth and proliferation. It has been found that $10 \mathrm{mV} \mathrm{DC}$ was beneficial to SCs. Overloading ES conditions may have inhibitory effects on the proliferation of SCs. These results indicated that the suitable ES on the conductive biomaterials might be a potential strategy to enhance SC proliferation and subsequently promotes PNI repair. It will provide more useful information for the development of PNI repair.

\section{Conflicts of interest}

There are no conflicts to declare.

\section{Acknowledgements}

This work was supported by the National Nature Science Foundation of China (grant 31571008).

\section{References}

1 A. Faroni, S. A. Mobasseri, P. J. Kingham and A. J. Reid, Adv. Drug Delivery Rev., 2015, 82-83, 160-167.

2 D. Grinsell and C. P. Keating, BioMed Res. Int., 2014, 2014, 698256.

3 X. Gu, F. Ding and D. F. Williams, Biomaterials, 2014, 35, 6143-6156.

4 M. P. Willand, M. A. Nguyen, G. H. Borschel and T. Gordon, Neurorehabil. Neural Repair, 2016, 30, 490-496.

5 M. Zhao, B. Song, J. Pu, T. Wada, B. Reid, G. Tai, F. Wang, A. Guo, P. Walczysko, Y. Gu, T. Sasaki, A. Suzuki, J. V. Forrester, H. R. Bourne, P. N. Devreotes, C. D. McCaig and J. M. Penninger, Nature, 2006, 442, 457-460. 
6 I. S. Aleem, I. Aleem, N. Evaniew, J. W. Busse, M. Yaszemski, A. Agarwal, T. Einhorn and M. Bhandari, Sci. Rep., 2016, 6, 31724.

7 J. Du, G. Zhen, H. Chen, S. Zhang, L. Qing, X. Yang, G. Lee, H. Q. Mao and X. Jia, Biomaterials, 2018, 181, 347-359.

8 A. N. Koppes, A. L. Nordberg, G. M. Paolillo, N. M. Goodsell, H. A. Darwish, L. Zhang and D. M. Thompson, Tissue Eng., Part A, 2014, 20, 494-506.

9 C. D. McCaig, A. M. Rajnicek, B. Song and M. Zhao, Physiol. Rev., 2005, 85, 943-978.

10 Y. Wu, L. Wang, B. Guo, Y. Shao and P. X. Ma, Biomaterials, 2016, 87, 18-31.

11 Y. Qian, X. Zhao, Q. Han, W. Chen, H. Li and W. Yuan, Nat. Commun., 2018, 9, 323.

12 J. Song, B. Sun, S. Liu, W. Chen, Y. Zhang, C. Wang, X. Mo, J. Che, Y. Ouyang, W. Yuan and C. Fan, Front. Mol. Neurosci., 2016, 9, 117.

13 H. Baniasadi, S. A. A. Ramazani and S. Mashayekhan, Int. J. Biol. Macromol., 2015, 74, 360-366.

14 L. Ghasemi-Mobarakeh, M. P. Prabhakaran, M. Morshed, M. H. Nasr-Esfahani, H. Baharvand, S. Kiani, S. S. Al-Deyab and S. Ramakrishna, J. Regener. Med. Tissue Eng., 2011, 5, e17-35.

15 T. Hanemann and D. V. Szabó, Materials, 2010, 3, 3468-3517. 16 X. Liu, C. Li, Y. Pan, D. W. Schubert and C. Liu, Composites, Part B, 2019, 164, 37-44.

17 M. Qu, F. Nilsson, Y. Qin, G. Yang, Y. Pan, X. Liu, G. Hernandez Rodriguez, J. Chen, C. Zhang and D. W. Schubert, Compos. Sci. Technol., 2017, 150, 24-31.

18 X. Wang, X. Liu, H. Yuan, H. Liu, C. Liu, T. Li, C. Yan, X. Yan, C. Shen and Z. Guo, Mater. Des., 2018, 139, 372-379.

19 N. Toshima, K. Oshima, H. Anno, T. Nishinaka, S. Ichikawa, A. Iwata and Y. Shiraishi, Adv. Mater., 2015, 27, 2246-2251.

20 T. Gordon, Neurotherapeutics, 2016, 13, 295-310.

21 A. N. Naik, S. Patra, D. Sen and A. Goswami, Phys. Chem. Chem. Phys., 2019, 21, 4193-4199.

22 J. Huang, X. Hu, L. Lu, Z. Ye, Q. Zhang and Z. Luo, J. Biomed. Mater. Res., 2010, 93, 164-174.

23 J. Y. Lee, C. A. Bashur, A. S. Goldstein and C. E. Schmidt, Biomaterials, 2009, 30, 4325-4335.

24 B. Sun, T. Wu, J. Wang, D. Li, J. Wang, Q. Gao, M. A. Bhutto, H. El-Hamshary, S. S. Al-Deyab and X. Mo, J. Mater. Chem. B, 2016, 4, 6670-6679.

25 Z. Zhang, L. H. Klausen, M. Chen and M. Dong, Small, 2018, 14, e1801983.

26 M. Joshi, B. Adak and B. S. Butola, Prog. Mater. Sci., 2018, 97, 230-282.

27 H. Liu, Y. Li, K. Dai, G. Zheng, C. Liu, C. Shen, X. Yan, J. Guo and Z. Guo, J. Mater. Chem. C, 2016, 4, 157-166.

28 G. Kaur, R. Adhikari, P. Cass, M. Bown, M. D. Evans, A. V. Vashi and T. Gunatillake, RSC Adv., 2015, 5, 9876298772.

29 H. Kim and S. Lee, Fibers Polym., 2017, 18, 1304-1313.
30 G. M. Laleh, M. P. Prabhakaran, M. Mohammad, N. E. Mohammad Hossein and R. Seeram, Tissue Eng., Part A, 2009, 15, 3605.

31 C. H. Jang, J. U. Lee and G. H. Kim, J. Ind. Eng. Chem., 2018, 64, 143-150.

32 W. C. Wang, Z. P. Wang, Y. Liu, N. Li, W. Wang and J. P. Gao, Mater. Res. Bull., 2012, 47, 2245-2251.

33 S. Meng, J. Tissue Eng. Regener. Med., 2014, 11, 274-283.

34 X. Wang, Y. Pan, C. Shen, C. Liu and X. Liu, Macromol. Rapid Commun., 2018, 39, e1800635.

35 W.-C. Wei, C. Deng, S.-C. Huang, Y.-X. Wei and Y.-Z. Wang, J. Mater. Chem. A, 2018, 6, 8643-8654.

36 Y. Qian, J. Song, X. Zhao, W. Chen, Y. Ouyang, W. Yuan and C. Fan, Adv. Sci. (Weinheim, Ger.), 2018, 5, 1700499.

37 J. Jin, Y. Lin, M. Song, C. Gui and S. Leesirisan, Eur. Polym. J., 2013, 49, 1066-1072.

38 Y. Tong, S. Bohm and M. Song, Appl. Surf. Sci., 2017, 424, 7281.

39 J. Wang, L. Tian, N. Chen, S. Ramakrishna and X. Mo, Mater. Sci. Eng., C, 2018, 91, 715-726.

40 J. Wang, Y. Cheng, L. Chen, T. Zhu, K. Ye, C. Jia, H. Wang, M. Zhu, C. Fan and X. Mo, Acta Biomater., 2019, 84, 98-113. 41 J. P. Lefaucheur, A. Antal, S. S. Ayache, D. H. Benninger, J. Brunelin, F. Cogiamanian, M. Cotelli, D. De Ridder, R. Ferrucci, B. Langguth, P. Marangolo, V. Mylius, M. A. Nitsche, F. Padberg, U. Palm, E. Poulet, A. Priori, S. Rossi, M. Schecklmann, S. Vanneste, U. Ziemann, L. Garcia-Larrea and W. Paulus, Clin. Neurophysiol., 2017, 128, 56-92.

42 K. Elzinga, N. Tyreman, A. Ladak, B. Savaryn, J. Olson and T. Gordon, Exp. Neurol., 2015, 269, 142-153.

43 R. Horn, Proc. Natl. Acad. Sci. U. S. A., 2005, 102, 4929-4930. 44 F. Bezanilla, Nat. Rev. Mol. Cell Biol., 2008, 9, 323-332.

45 M. Bikson, M. Inoue, H. Akiyama, J. K. Deans, J. E. Fox,

H. Miyakawa and J. G. Jefferys, J. Physiol., 2004, 557, 175-190. 46 A. N. Koppes, A. M. Seggio and D. M. Thompson, J. Neural Eng., 2011, 8, 046023.

47 J. Zhang, K. Qiu, B. Sun, J. Fang, K. Zhang, H. Ei-Hamshary, S. S. Al-Deyab and X. Mo, J. Mater. Chem. B, 2014, 2, 79457954.

48 T. Gordon and A. W. English, Eur. J. Neurosci., 2016, 43, 336350.

49 J. Yao, H. Wang, M. Chen and M. Yang, Mikrochim. Acta, 2019, 186, 395.

50 S. Syama and P. V. Mohanan, Int. J. Biol. Macromol., 2016, 86, 546-555.

51 M. Ema, M. Gamo and K. Honda, Regul. Toxicol. Pharmacol., 2017, 85, 7-24.

52 Y. Chang, S. T. Yang, J. H. Liu, E. Dong, Y. Wang, A. Cao, Y. Liu and H. Wang, Toxicol. Lett., 2011, 200, 201-210.

53 K. H. Liao, Y. S. Lin, C. W. Macosko and C. L. Haynes, ACS Appl. Mater. Interfaces, 2011, 3, 2607-2615. 